\title{
Topology and Differential Geometry-based Three-dimensional Characterization of Pearlite Spheroidization
}

\author{
Yuan-Tsung WANG, ${ }^{1)}$ Yoshitaka ADACHI ${ }^{21}$ Kiyomi NAKAJIMA ${ }^{1)}$ and Yoshimasa SUGIMOTO ${ }^{1)}$ \\ 1) National Institute for Materials Science, Innovative Materials Engineering Laboratory, 1-2-1 Sengen, Tsukuba, Ibaraki, 305- \\ 0047 Japan. $\quad 2$ 2) Foremerly at National Institute for Materials Science, Innovative Materials Engineering Laboratory. Now \\ at Department of Mechanical Engineering, Graduate School of Science and Engineering, Kagoshima University, Korimoto 1-21- \\ 24, Kagoshima, 890-8580 Japan. E-mail: adachi@mech.kagoshima-u.ac.jp
}

(Received on February 18, 2011; accepted on August 23, 2011)

\begin{abstract}
Spheroidized pearlite microstructures in three-dimensions (3D) in a 0.8 mass $\% \mathrm{C}-\mathrm{Fe}$ steel under $700^{\circ} \mathrm{C}$ static annealing was examined focusing on topology and differential geometry. The topological or differential-geometric features of the microstructures were examined by evaluating the genus and the Gaussian/mean curvatures. 3D visualization demonstrated that the holes intrinsic in cementite lamellae are significant morphological features affecting the kinetics of the initial pearlite spheroidization, due to the mean curvature differences between the hole edge and the adjacent flat surface. The hole coalescence and expansion influence not only the morphological evolution, but also the topological characteristics. The genus per unit volume decreases at an earlier spheroidization time due to a decrease in the number of holes and an increase in independent bodies, but increases at longer coarsening time because of a decrease in independent bodies. The distribution area of nonzero probability of the mean and Gaussian curvature decreases with an increase in spheroidization time, which agrees with the increase in the length scale of the microstructures.
\end{abstract}

KEY WORDS: cementite lamellar; Gaussian curvature; mean curvature; topology; genus; spheroidization.

\section{Introduction}

Two-phase lamellar structures are commonly observed in metallic systems, e.g., eutectoid pearlite and eutectic alloys. Based on two-dimensional (2D) observation, several models for the mechanism of spheroidization of lamellar structures have been proposed ${ }^{1-3)}$ however, no direct measurement of the topological and curvature evolution of the lamella interface by quantitative three-dimensional (3D) analysis has been reported for different spheroidization periods. 3D image analysis provides a deeper insight into the spheroidization mechanism of lamellar structures, as it allows the measurement of the local interface curvature and topology for spheroidized microstructures quantitatively, which is not available from $2 \mathrm{D}$ observations. While the kinetics of pearlite spheroidization and the morphological evolution can be quantified by measuring the probability of locating a patch of interface with a given curvature tensor, $\left.{ }^{4}\right)$ or surface area per unit volume $\left(\mathrm{S}_{\mathrm{v}}\right),{ }^{5)}$ additional information can be obtained by measuring the topological properties of the 3D image, such as the genus. Recently, through developments in the manner in which serial sections are collected and in the methods used to reconstruct the 3D morphology of the serial sectioning images, it is possible to gain new insights into the manner in which pearlite spheroidization occurs. In a previous paper, ${ }^{6,7)}$ it was demonstrated based on a 3D image that there were many morphological faults such as a hole and a fisher in cementite lamellae. Similar morphological faults were also observed by other researchers. ${ }^{8)}$

In this study, we use computer-aided $3 \mathrm{D}$ visualization to investigate the influence of surface curvature variation due to the existence of microstructural faults on the morphological evolution of cementite lamellae during static annealing, and to clarify the elements contributing to topological variations of pearlite by obtaining the genus of microstructures at different spheroidization stages.

\section{Experimental Procedure}

The chemical composition of the steel used in this study was 0.8 mass $\% \mathrm{C}-\mathrm{Fe}$. Hot-rolled steel specimens were reaustenitized at $1200^{\circ} \mathrm{C}$ for $30 \mathrm{~min}$, followed by cooling in the furnace to room temperature at $1^{\circ} \mathrm{C} / \mathrm{s}$. Subsequently, the specimens were isothermally annealed at $700^{\circ} \mathrm{C}$ for 0,60 , and $360 \mathrm{~min}$, followed by furnace cooling at $1^{\circ} \mathrm{C} / \mathrm{s}$ to room temperature. For the 3D tomography observation, a doublebeam SEM (SII-Zeiss XVision200DB) was used, where serial sectioning by focused ion beam (FIB) and SEM observation were alternatively and repeatedly conducted in an automatic operating mode. The sectioning interval was 100 $\mathrm{nm}$, and about 92 total sections existed for each condition. Prior to FIB-SEM operation, $5 \times 3 \times 3 \mathrm{~mm}^{3}$ specimens were mechanically polished on the two sections perpendicular to each other, to achieve a sharp edge and a damage-free surface. The 3D microstructures were reconstructed by stacking and alignment of the serial sectioning images and the 
appropriate crystallographic orientation assigned to the section. Avizo and MAVI express software packages provided all image processing, visualization, and microstructural and quantitative analysis. ${ }^{9)}$

\section{Analysis}

\subsection{Surface Curvature}

The driving force behind spheroidization of a lamellar structure is the reduction of the surface energy by minimizing the surface area per unit volume. This is because there is excess free energy associated with the presence of the surface. The surface area is reduced through a mass diffusion process from regions of high surface curvature to regions of low surface curvature, in which the total surface area is reduced over time. Two parameters can be used to characterize the surface curvature: the mean curvature $\mathrm{H}$ and the Gaussian curvature $\mathrm{K}$, given as: ${ }^{10)}$

$$
\begin{array}{r}
\mathrm{H}=\frac{1}{2}\left(\frac{1}{\mathrm{R}_{1}}+\frac{1}{\mathrm{R}_{2}}\right)=\frac{1}{2}\left(k_{1}+k_{2}\right) \\
\mathrm{K}=k_{1} \times k_{2} \ldots \ldots \ldots \ldots \ldots \ldots
\end{array}
$$

where $k_{1}$ and $k_{2}$ are the two principle curvatures of the maximum and minimum values among all normal curvatures and are defined as $k_{1}=1 / \mathrm{R}_{1}$ and $k_{2}=1 / \mathrm{R}_{2}$, where $\mathrm{R}_{1}$ and $\mathrm{R}_{2}$ are the principal radii of curvature that are tangent to the surface patch. The significance of the variation in the surface mean curvature for this mass transfer to occur, and the equilibrium matrix concentration of lamellar structure $(C)$ can be described by the Gibbs-Thomson equation for a binary alloy: ${ }^{11}$

$$
\mathrm{C}=\mathrm{C}_{\mathrm{o}}\left[1+\frac{2 \gamma_{s} V}{r R T}\right]=C_{o}+\alpha H
$$

where $\mathrm{C}_{0}$ is the equilibrium matrix concentration of the lamellar material, $\gamma_{s}$ the surface energy, $V$ the molar volume of the lamellar phase, $r$ the radius of the interface, $R$ the gas constant, $T$ the temperature, $\alpha$ represents the quantity $\mathrm{C}_{\mathrm{o}} \gamma_{\mathrm{s}} V /$ $R T$, and $\mathrm{H}$ is the mean curvature of the surface. As seen from Eq. (3), the variation in curvature with position results in concentration gradients, which leads to mass fluxes from high mean curvature regions to low ones. It is important to note that these mass transportations occur at essentially a constant volume condition. ${ }^{12)}$ As the material is deposited or removed, the mean curvature will change. Although only the local mean curvatures influence the transport of mass via diffusion, the local mean curvature at any instant is not independent of the local Gaussian curvature. ${ }^{13)}$ The importance of the Gaussian curvature is that it can provide an essential measure of the morphology and express the characterization of the interface. According to Eq. (3), a plot showing the surface mean curvature can directly reveal the locations of dissolution and deposition of the solute, but a precise description of the mass flux requires to take large regions of the structure into consideration. Therefore, a more quantitative representation of large regions of the surface curvature data can be given by plotting the probability distribution of mean and Gaussian curvature. Figure 1 shows the map of the different surface-shape contours for the mean and Gaussian curvature plot (H-K plot). This map provides us with the following information:
- Solving Eqs. (1) and (2), provides the relationship $\mathrm{H}^{2}-$ $\mathrm{K} \geq 0$. Curvatures that lie on the parabola described by $\mathrm{H}^{2}-\mathrm{K}=0$ correspond to spherical local surface shapes, as shown in Fig. 1. If $\mathrm{H}$ is positive, the surface has the spherical shape of cementite $(\theta)$; if $\mathrm{H}$ is negative, the surface has the spherical shape of ferrite $(\alpha)$. Furthermore, if $\mathrm{H}=0$ and $\mathrm{K}=0$, the surface is locally flat or planar.

- If a point on the surface has a positive Gaussian curvature, the concavity of the surface is determined by the sign of $\mathrm{H}$. If $\mathrm{H}$ is positive, the surface is concave toward the cementite; if $\mathrm{H}$ is negative, it is convex toward the cementite.

- An interface with $\mathrm{K}=0$ has a cylindrical surface composed of cementite (if $\mathrm{H}$ is positive) or ferrite (if $\mathrm{H}$ is negative) and it is also equal to the cylindrical cementite within planar ferrite or cylindrical ferrite within planar cementite.

- Saddle-shaped surfaces are characterized by a negative Gaussian curvature.

Therefore, in this study, the complex surface geometries created in cementite lamellae during spheroidization will be quantified with the probability distribution of the mean and Gaussian curvature (so-called H-K plot).

\subsection{Surface Topology}

The topology of 3D surfaces is related to the volume integral of the Gaussian curvature $\left(K_{\text {total }}\right)$ by the Gauss-Bonnet theorem, ${ }^{10)}$ and can be expressed by Eq. (4):

$$
g=1-\chi_{3}=1-\frac{K_{\text {total }}}{4 \pi}
$$

where $g$ and $\chi_{3}$ are the genus and the Euler characteristic, respectively. These quantities depend only on the topology of the surface and not on its shape. For instance, the genus represents the number of holes in the surface, provided that the surface is closed. If a sphere and any other polyhedron have no holes in the closed surface, the integral Gaussian curvatures $\left(K_{\text {total }}\right)$ are equal to $4 \pi$ and they have a genus of zero. Attaching a hole to the structures decreases $K_{\text {total }}$ by $4 \pi$ and increases the genus by one. However, if the curvature information of a surface is not available, the Euler characteristic can also be obtained by triangulating the 3D surface $^{14)}$ and then counting the number of nodes n, edges e, faces $\mathrm{f}$, and cells (polygon) $\mathrm{c}$ as expressed in Eq. (5):

$$
\chi_{3}=1-g=n-e+f-c
$$

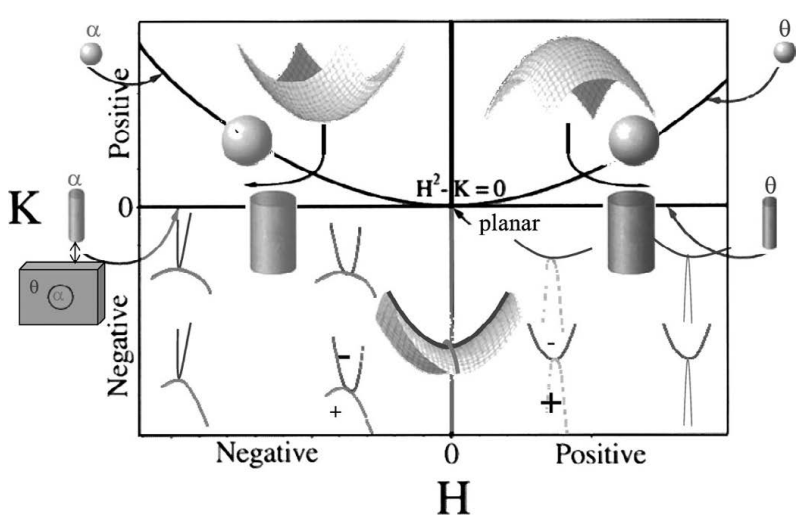

Fig. 1. Map of local surface shapes for the H-K contour plots. 
(a)

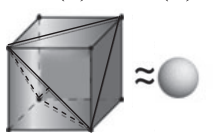

(f)

(b)

\section{O}

(g)

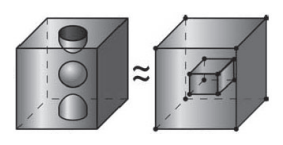

(c)

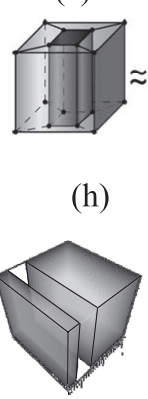

(d)

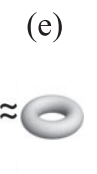

(i)

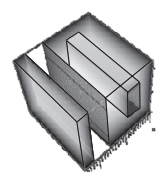

Fig. 2. Topologically equivalent shape.

Thus, for a simple cube or a solid body, the structure is topologically equivalent to a sphere (Figs. 2(a), 2(b)) and $g=0$, resulting in $\chi_{3}=1$. However, if the cube or the body is not closed, $\chi_{3} \neq 1$ and Eq. (5) must be used to determine $\chi_{3}$. For example, if a square hole or a tunnel goes completely through a cube (Figs. 2(c), 2(d)), the results are $n=16, e=36$, $f=24$, and $c=4$, giving $\chi_{3}=0$ and $g_{\mathrm{v}}=1$. Therefore, the shape would be topologically equivalent to a torus (Fig. 2(e)).

In addition to the quantitative measurement of the microstructure connectivity, the genus can also express shape instabilities, such as the breakup of cylindrical (Figs. 2(f), 2(g)) or long narrow ribbon structures (Fig. 2(h)) through Rayleigh or surface energy instability, respectively. ${ }^{15,16)}$ Therefore, the morphological instability of the cylinder hole or tunnel, as illustrated in Fig. 2(d), eventually decomposes into a row of spheres (Fig. 2(f)), a completely enclosed void is created, and the structure is topologically equivalent to a cubic void within a cube (Fig. 2(g)), where $n=16, e=32, f=24$, and $c=6$, resulting in $\chi_{3}=2$ and $g=-1$. In these cases, the topological characterization of holes or voids within only one independent body can be properly expressed by Eq. (5); however, it must also consider the number of independent bodies for application to topologically complex systems. It has been reported that intrinsic holes play an important role in the breakup of large cementite lamellae into several long narrow ribbons during spheroidization. ${ }^{16)}$ For example, a cementite lamella is decomposed into two single bodies by hole expansion (Fig. 2(h)), where $n=16, e=36, f=32$, and $c=10$. We put the result into Eq. (5) and it can obtain $\chi_{3}=2$ and $g=-1$. Therefore, the morphological instability of the structural decomposition decreases the genus by 1 . Also, it is evident that a structure with a single void has a genus of -1 . Thus, both cases decrease the genus by one, but creating a hole to the structure increases the genus by one. Provided that there is a relationship among $g, h, b$, and $v$, the genus (g) can be expressed by Eq. (6):

$$
g=h-(b+v)+1
$$

where $h, b, v$ are the numbers of hole, independent body and void, respectively. This equation is applicable to both general and complex topological material systems. For instance, if there is one body, genus is given by $g=h-(1+v)+1=h-v$. $\mathrm{h}$ and $v$ correspond a continuous second phase and discontinuous second phase embedded in a matrix phase. This means in general that $g$ can be used as a finger print how continuous the second phase is. However, if $b>v$, the meaning of $g$ becomes more complex and a parameter to adjust
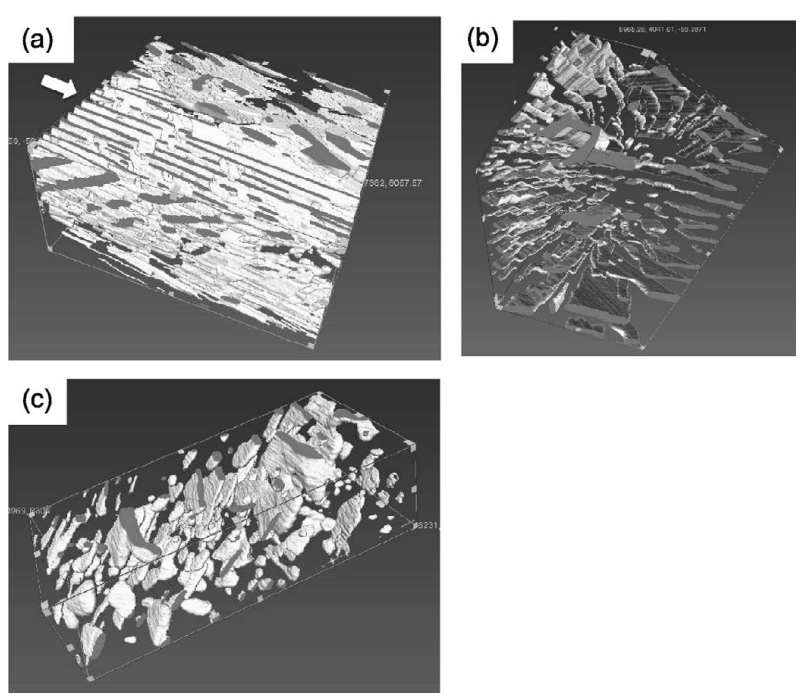

Fig. 3. 3D reconstructed images of (a) full cementite and annealing at $700^{\circ} \mathrm{C}$ for (b) 60 and (c) $360 \mathrm{~min}$, respectively.

the relationship among $h, b$ and $v$. If there are two bodies, adding a tunnel to one of the two bodies (Fig. 2(i)), $h=1$, $b=2$, and $v=0$, gives $g=0$ and $\chi_{3}=1$. This result is in good agreement with that calculated by Eqs. (4) and (5). In this study, we combine the Euler characteristic (Eq. (5)) and genus (Eq. (6)) to demonstrate the topological characterization of pearlite spheroidization. The geometric surfaces of cementite and ferrite phases created at different spheroidization stages, i.e. independent bodies and voids, can be triangulated and examined by calculating the number of nodes, edges, faces, and cells using MAVI, a computer-aided software. Then, the quantitative analysis of the topological characteristics is accomplished by examining the genus and the numbers of independent bodies, voids, and holes.

Another length scale required to understand the evolution of topologically lamellar structures over a long spheroidization time is the coarsening process. The length scale parameter used here is the inverse of the surface area per unit volume, $1 / S_{v}$, and is related to the spheroidization time, and can be expressed as in Eq. (7):

$$
S_{v}^{-3}(t)-S_{v}^{-3}(0)=K t
$$

Because the mass flux is a result of the spatially varying surface curvature, the geometric morphology including the corresponding topological characteristics is required for full understanding of the spheroidization and coarsening processes. Therefore this study examines a variation of the surface curvature and topology of cementite with annealing time.

\section{Results}

A change of the 3D cementite microstructures with annealing time is demonstrated in Fig. 3. In the as-patented condition, the cementite microstructure exhibits the typical lamellar structure of full pearlite, as shown in Fig. 3(a). After annealing, the longitudinal shape of cementite lamellae had gradually changed shape and evolved into smaller shapes with increasing annealing time (Fig. 3(b)). They form a partially spheroidized structure after spheroidization heat treatment at $700^{\circ} \mathrm{C}$ for $360 \mathrm{~min}$ (Fig. $3(\mathrm{c}))$. To understand the complex geometric evolution of 
cementite lamellae during spheroidization in detail, the variation of the mean and Gaussian curvature of 3D microstructures was examined, as shown in Fig. 4, where the surface is colored based on the local mean and Gaussian curvature. The images illustrate that a real pearlitic lamellar structure is never perfect and many holes exist in it (Fig. 4 (a)). ${ }^{6,7)}$ The regions surrounding the holes have high mean curvatures (red or yellow) in the lamellar structure compared with the near flat plane (green), where it may be considered thermodynamically equivalent to planar surfaces, $\mathrm{H}=\mathrm{K}=0$, and so do not provide a thermodynamic motivation for shape change or interface motion. As illustrated in Eq. (3), this non-uniform curvature leads to concentration gradients, which in turn excite mass fluxes and diffusions. On annealing, the curvature difference between the hole edges and the adjacent flat surface of the lamella induces mass transfers from the high mean curvature regions of the hole edge to low ones of the flat interface, resulting in rapid dissolution of the hole edge and thickening of the adjacent flat surface of the ribbon-like bulges (Fig. 4(b), labeled B). During this process, the total surface area decreases and the driving force for hole growth is obtained from the surface energy reduction. As long as curvature difference exists, the mass flow from the hole edge to the adjacent flat or low curvature plane is sustained, and the large cementite lamellae can break up into several long narrow ribbons during the initial spheroidization.

The sequence of events subsequent to cylinderization or further spheroidization formation depends on the ribbon's cross- sectional aspect ratio. The progression in shape instability during static annealing is schematically illustrated in
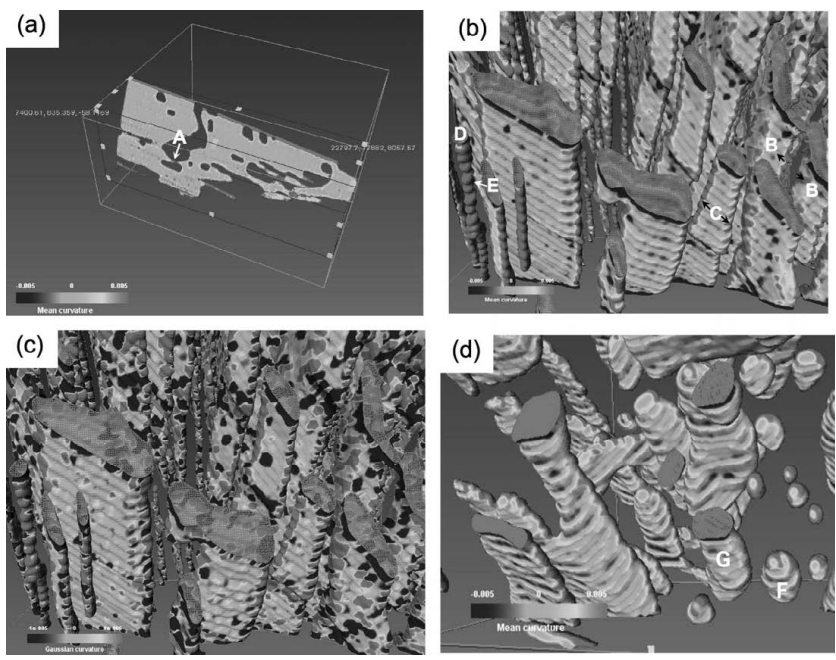

Fig. 4. Curvature images of cementite (a) before and after spheroidization at $700^{\circ} \mathrm{C}$ for (b, c) 60 and (d) $360 \mathrm{~min}$. (b) and (c) are mean and Gaussian curvature images, respectively.
Fig. 5, using a finite cylinder with a semi- cylindrical edge. In high aspect ratio conditions (Figs. 5(a), 5(b)), the shape transformation results from the mean curvature difference between the ribbon edge and the adjacent flat surface of the ribbon, as shown in Fig. 4(b). This transport causes the ribbon edges to recede and produces a buildup of material on the flat surface immediately adjacent to the edge (Fig. 5(c)). The continuation of edge recession to produce cylinders indicates that the geometry evolves to maintain a driving force for mass transport from high curvatures of ribbon edges to lower curvatures of inner surfaces. When edge diffusion fields overlap, the final equilibrium cylindrical shape is approached (Fig. 5(d)). Subsequent to cylinderization, the cylinder eventually decomposes into a row of spheres (Fig. 5(f)), via Rayleigh's perturbation process, after long period of spheroidization. ${ }^{16)}$

Cylinderization, which occurs in ribbons of low aspect ratio, also results from continued edge recession. However, if the aspect ratio is very low, or its morphology is very closed to long cylinders (Fig. 4(b) labeled D), the spheroidization may proceed directly (Figs. 5(a), 5(d)). An earlier investigation on pearlite spheroidization showed that the disintegration of pearlite ribbons into smaller segments by surface energy reduction is possible without previous transformation of the ribbons into cylinders. ${ }^{17)}$ As shown in the Gaussian curvature (Fig. 4(c)), the boundary surface will transfer to a bumpy one during spheroidization. For more detailed identification of the low aspect-ratio and cylindrical shape of cementite beadroll (Fig. 4(b) labeled D), the high mean curvatures are present at thin necks of the spherical particles (Fig. 4(b) labeled E, dark red color) compared with the other portions of the cementite beadroll. The mean curvature difference leads to mass transportation from the neck base of the spherical particle and to accumulation at the region near the tip of spherical particle (Fig. 5(e)), therefore decreasing $\mathrm{R}_{1}$ and increasing $R_{2}$. If these trends continue for longer spheroidization periods, the cylinder would evolve to an array of spherical particles, as shown in Fig. 4(b) labeled D. When the diameter of the spherical particles near the tips continues to grow until $\mathrm{R}_{1}$ becomes zero, the spherical particles detach from the cylinder (Fig. 5(f)). On further annealing, these segments can break up into many small spheres (Fig. 4(d)).

\section{Discussion}

As mentioned above, the curvature variations and corresponding mass concentration variations lead to fluxes of solute from high mean curvature regions to low ones. However, the surface interactions due to the curvature difference change not only the microstructure geometry, but also the topological characteristics. Complete understanding of the

Increasing time

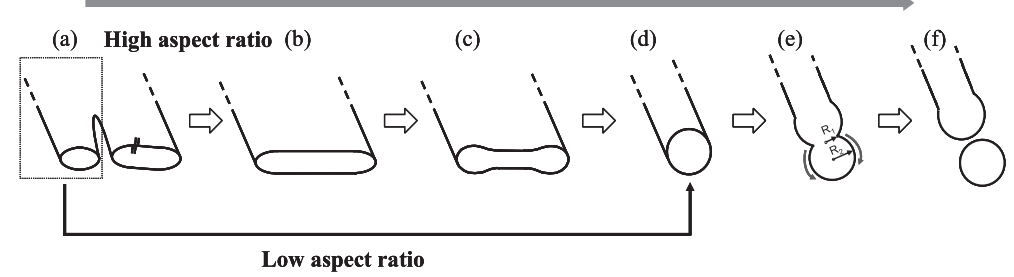

Fig. 5. Schematic of the progression in shape instabilities of cementite spheroidization during static annealing. 
evolution of cementite spheroidization and the coarsening process requires quantitative analysis of the spatial variation of surface curvature and topology as a function of annealing time. The topologies of pearlite structures are characterized by examining the geometric surface evolution of 3D images obtained at different spheroidization stages. In this study, a hole is defined as a tunnel completely piercing the cementite lamellae. The number of independent bodies corresponds to the number of cementite lamellae decomposed by hole coalescence or expansion, and a void is a isolated hole completely enclosed by ferrite. To remove volume effects, these topological analyses are reported on a unit volume basis. The number of holes per unit volume $\mathrm{h}_{\mathrm{V}}$, is found to decrease with an increase in annealing time as shown in Fig. 6. These holes play an important role in the initiation of pearlite spheroidization. This is due to the existence of a curvature difference between the interface of hole edges and the adjacent flat surfaces of the cementite lamellae, which indicates that excess free energy is localized at the surface. ${ }^{18)}$ The necessity to minimize the extra surface energy makes cementite lamellae thermodynamically unstable. Careful examination of many holes in Fig. 4(a) revealed that the majority of red high mean curvature are located in holes inside and on the neck of the bridge between holes (labeled A in Fig. 4(a)), illustrating that the curvature difference between the hole edges and the adjacent flat surface of the lamella drives this shape transformation. It has been reported that at this instant, if two holes are very close, Rayleigh's perturbation theory dominates and continues the break-up of the bridges until the coalescence of holes. ${ }^{19)}$ Thus, these holes in cementite lamellae will increase in size with increasing spheroidization time. It appears as if two holes have coalesced and expanded, leading to a decrease in the surface area that causes the breakup of large cementite lamellae into several long, narrow ribbons to decrease the difference in surface energy. This is the reason why the number of holes per unit volume decreases with an increase in the annealing time, as shown in Fig. 6. The cementite spheroidization probably proceeds mainly through the growth of holes in cementite lamellae on relatively early stage. In addition, the edge of initial lamellar cementite can also contribute to accelerate cementite spheroidization, if total surface area decreases. Which can accelerate spheroidization

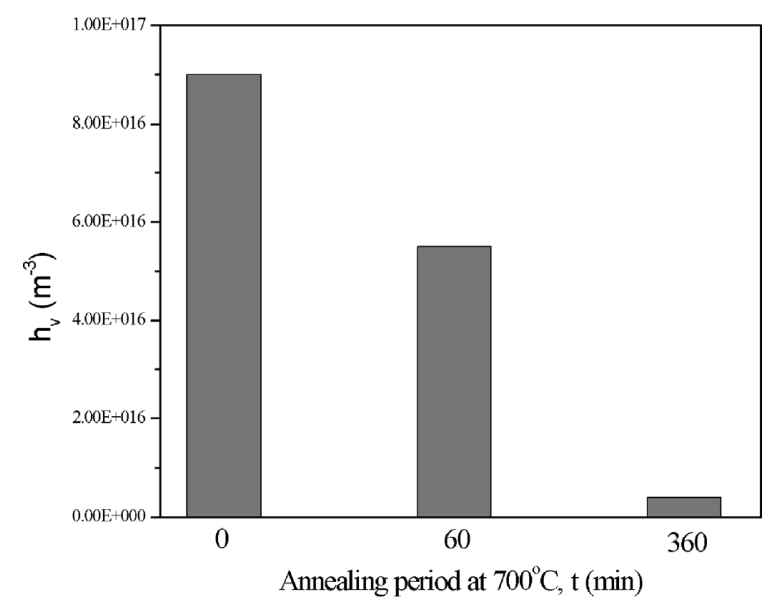

Fig. 6. Number of holes per unit volume as a function of spheroidization time at $700^{\circ} \mathrm{C}$. much more, the edge or the hole, largely depends on absolute value of mean or main curvature.

Although the driving force for holes to expand in cementite lamellae is the surface area reduction, the formation of new surface area is followed by the separation of the large cementite lamellae into several long, narrow ribbons. Therefore, the total surface area of the longitudinal boundaries of narrow ribbons must increase and the height mean curvatures are present where the longitudinal edges are formed from large cementite lamellae (Fig. 4(b), labeled C). The curvature difference between the ribbon edge and the adjacent flat surface of the ribbon will accelerate the spheroidization of pearlite and eventually break it up into a ring of spheres after long spheroidization periods, as shown in Fig. 4(d). Since the mean curvature of a sphere is given by $\mathrm{H}=1 / \mathrm{R}$, according to Eq. (3), the concentration at the surface of small particles will be higher than that for large particles. Thus, over time, the radius of the small particles will decrease, while the radius of large spherical (Fig. 4(d), labeled F) or cylindrical-like (labeled G) particles will increase, because the shape having a small surface area per unit volume corresponds to the large particles. Throughout these changes, the total volume fraction of cementite remains constant. The average particle size increases with time, while the number density of particles decreases. To quantify the increase in the length scale of the microstructures, the inverse surface area per unit volume is calculated for each annealing condition. Figure 7 shows that a linear fit is in good agreement with the data, which means that even after the dramatic morphological change from lamellar structures to the ones consisting of spherical particles has taken place (Fig. 3), $S_{v}^{-3}$ is still linearly related to time. This means that the surface area per unit volume $S_{v}$ decreases over time and some small particles decomposed from cylinder, via Rayleigh's perturbation mechanism, start to coarsen by Ostwald ripening for long spheroidization period. ${ }^{20)}$ Throughout these changes, the average particle size increases with time, while the total surface area and hence, the total surface energy decreases.

Since the mass fluxes are a result of a spatially varying surface curvature and geometry, a 3D image showing the interface colored according to the mean curvature, i.e., Fig. 4(a), directly reveals the possible locations of dissolution (high mean curvature) and deposition of mass (low or

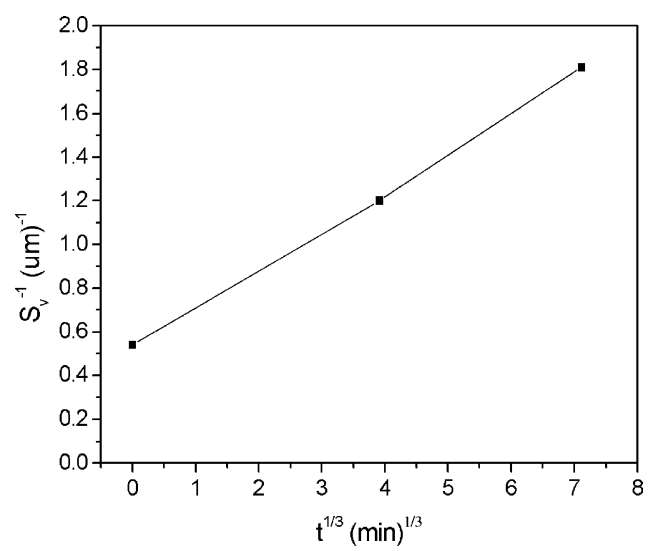

Fig. 7. $\mathrm{S}_{\mathrm{v}}{ }^{-1}$ as a function of the cube root of spheroidization time, for the three condition specimens. 
(a)
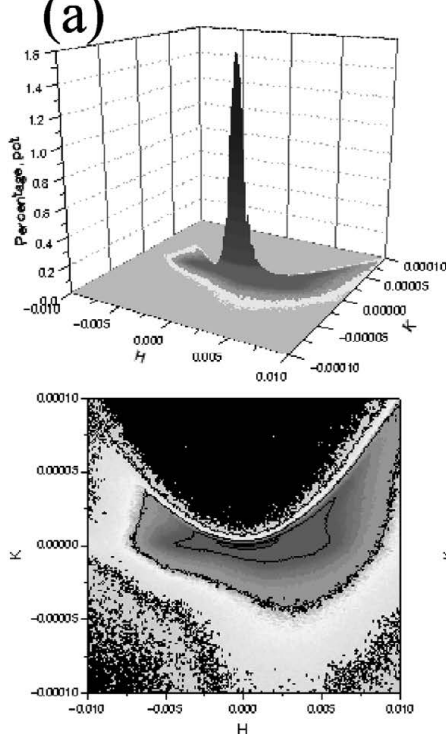

(b)
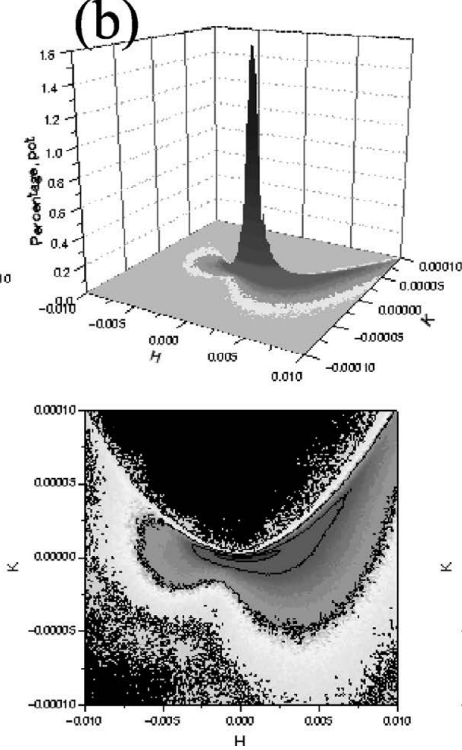

(c)
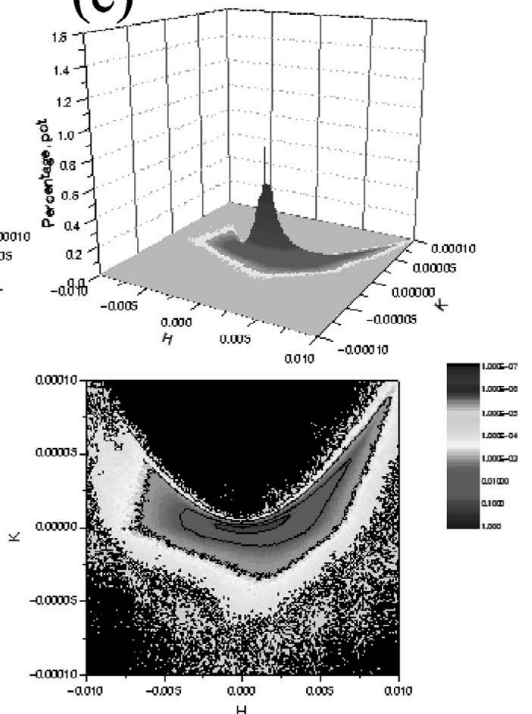

Fig. 8. Plots of distribution characterizations of mean and Gaussian curvature as a function of spheroidization time at $700^{\circ} \mathrm{C}$ for (a) 0 , (b) 60 and (c) $360 \mathrm{~min}$, respectively.

negative mean curvature). However, the rate of this process is not determined completely by the mean interfacial curvature at a point, but is also influenced by the curvature distribution along large regions of the surface surrounding this point, since these regions of the interface communicate via a long-range diffusion field. ${ }^{21)}$ To understand the complex topological evolution of the cementite structures during spheroidization and coarsening process, the distribution of the mean and Gaussian curvature, divided by all of the curvature data in the volume, as a function of spheroidization time, are examined and shown in Fig. 8. The distribution area of nonzero probability of the H-K plot decreases with an increase of annealing time, which agrees with the increase in the length scale of the microstructures, shown in Fig. 7. For a full pearlite specimen (Fig. 8(a)), the surface patches dispersed through the microstructure are the prevalent shape, indicating that the $3 \mathrm{D}$ microstructure of cementite has very complex topology.

Under annealing at $700^{\circ} \mathrm{C}$, the distributions narrow and the curvature values tend to decrease, indicating that the surface evolves through a path of decreasing free energy. It is interesting to find the morphology with negative Gaussian curvature $(<0)$ in Fig. $8($ b). Compared to the result shown in Fig. 8(a), the intensity of $\mathrm{H}-\mathrm{K}$ plot with negative Gaussian curvature is slightly shrunk and this is greatly enhanced with an increase in annealing time. This is because many holes have coalesced and grown at this stage (Fig. 6) and caused the breakup of large cementite lamellae into several long, narrow ribbons to decrease the difference in surface energy, or the curvature between hole edges and the adjacent flat surface of the lamella. It is clear from Figs. 4(b), 4(c) that round-edged ribbon shapes dominate the structure, accompanied by a few cylindrical-like shapes or spherical particles, which stretch along the $\mathrm{H}^{2}-\mathrm{K}=0$ line. With an increase in annealing time (Fig. 8(c)), the fraction of surfaces with extreme positive and negative mean and Gaussian curvatures decrease, approaching relatively low $\mathrm{H}$ and $\mathrm{K}$ reagion. This result confirms the fact that the surface evolves toward a more stable state by minimizing its curva-

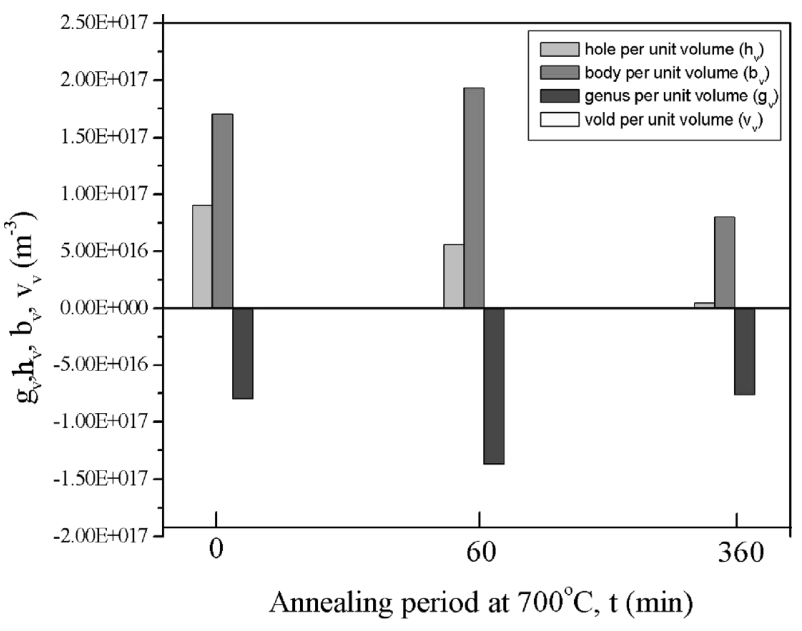

Fig. 9. Number of genus, holes, independent bodies, and voids per unit volume as a function of spheroidization time at $700^{\circ} \mathrm{C}$.

ture. On observing the microstructure of this specimen, it is concluded that the average particle size increases, while the number density of particles decreases, as shown in Fig. 4(d). The net effect of this process is an increase in the average length scale of the microstructure and a corresponding decrease in the average surface curvature. The overall result is that the microstructure evolves into coarser structures.

To quantitatively analyze the connectivity of the geometric surface evolution of the microstructure, the genus is characterized as a function of annealing time by examining the number of holes, independent bodies and voids, as illustrated in Eq. (6). The results show that the genus per unit volume decreases at an earlier spheroidization time, but increases at a longer coarsening time, as shown in Fig. 9. By reconstructions of the $3 \mathrm{D}$ microstructures of cementite and ferrite phases using MAVI software (Fig. 10) and calculating the number of ferrite body in the volume examined, the result shows that for all conditions, the number of ferrite body is one. This means that there are no voids in cementite. According to Eq. (6), a decrease in the genus is a result of a decrease in the number of holes, an increase in the number 

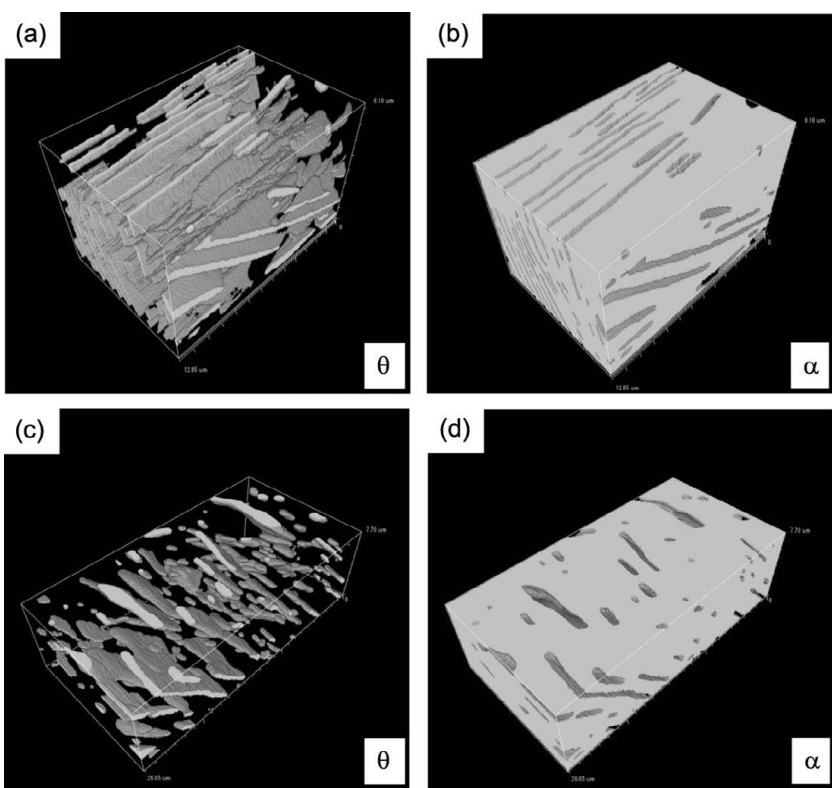

Fig. 10. Reconstructions of the $3 \mathrm{D}$ microstructures of cementite and ferrite phases at $700^{\circ} \mathrm{C}$ for (a, b) 0 and (c, d) $360 \mathrm{~min}$, respectively, using MAVI software.

of independent bodies and voids, or a combination of both. Because the number of voids per unit volume is zero, $v_{v}=0$, it is possible to separate the hole and independent body effects. The largest contributor to the decrease in $g_{v}$ at early spheroidization times seems to be the decrease in holes and increase in independent bodies. However, the number of holes and independent bodies per unit volume decrease with an increase in spheroidization time. Compared with the earlier spheroidization time, the decrease in amplitude of the $b_{v}$ values is more evident than that of $h_{v}$. Thus, the decrease in $b_{v}$ becomes a larger contributor to the increase in $g_{v}$ at later coarsening times. At this stage, although the genus is increased, its value is still negative. Mendoza et al. ${ }^{22)}$ reported that it is possible for the genus of a microstructure to become negative if the number of bodies or voids is greater than the number of holes. The reason of the variation in cementite body number can be explained as follows. At the beginning of spheroidization, a sheet cementite lamella is disintegrated into ribbon shape and then the cementite changes their shape from ribbon one to rod one. Furthermore rod-shaped cementite lamellae transform to sphere-shaped one. During these stages, indeed, the number of cementite body likely increases. However, to reduce surface energy, coalescence of small cementite particles concurrently takes place during relatively long annealing, which leads reduction in number of cementite bodies. Actually these two opposing phenomena proceed simultaneously, so that a maximum in the number of cementite body appears at middle annealing period.

The surface curvature and topological analysis of pearlite microstructures suggest that the holes present in cementite lamellae decrease with increasing annealing time, so that it is plausible to consider that morphological defects such as holes or fissure affect the kinetics of the pearlite spheroidization.

\section{Summary}

The variation of 3D morphologies of cementite lamella in eutectoid pearlitic steel during spheroidization was examined intensively, focusing on topology and differential geometry. The shape and energy instability induction of morphological evolution of the 3D lamellar structure during spheroidization were discussed. 3D visualization demonstrated that the intrinsic holes present in cementite lamella likely accelerate pearlite spheroidization. The curvature difference between the hole edge and the adjacent flat surface induces the breakup of large cementite lamella into several long, narrow ribbons to decrease the surface area and the energy during the initial spheroidization stage. These cementite ribbons can change their shape to cylinders or small particles, due to the curvature differences between the ribbon edge and the adjacent flat surface of the ribbon after prolonged spheroidization period. The number of holes actually decreases with increasing annealing period according to topology and differential geometry analyses. The genus per unit volume decreases at earlier spheroidization time, due to the decrease of holes and increase in independent bodies, but increases at longer coarsening times because of the decrease in independent bodies. The distribution area of nonzero probability of the mean and Gaussian curvature decreases with the increase in spheroidization time, which agrees with the increase in the length scale of the microstructures.

\section{Acknowledgements}

All authors express their gratitude to the National Institute for Materials Science, Japan for supporting this study. We acknowledge Professor Peter Voorhees, Northwestern University for fruitful discussion on topological view point. One of the authors (Y. T. Wang) gratefully acknowledges the financial support of the China Steel Corporation of Taiwan, ROC.

\section{REFERENCES}

1) J. C. Malzahn Kampe, T. H. Courtney and Y. Leng: Acta. Metall., 37 (1989), 1735.

2) F. A. Nichola and W. W. Mullins: Trans. AIME, 233 (1965), 1840

3) E. A. Chojnowski and W. J. Tegart: Met. Sci. J., 2 (1968), 14.

4) R. Mendoza, I. Savin, K. Thornton and P. W. Voorhees: Nat Mater, 3 (2004), 385.

5) S. Marsh and M. Glicksman: Metall. Mater. Trans. A, 27A (1996), 557.

6) Y. Adachi, S. Morooka, K. Nakajima and Y. Sugimoto: Acta Mater., 56 (2008), 5995.

7) Y. T. Wang, Y. Adachi, K. Nakajima and Y. Sugimoto: Acta Mater., 58 (2010) 4849.

8) M. D. Graef, M. V. Kral and M. Hillert: J. Minerals., 58 (2006), 25.

9) Y. Adachi: Bull. Iron Steel Inst. Jpn., 16 (2010), 28.

10) A. Gray, E. Abbena and S. Salamon: Morden Differential Geometry of Curves and surface with Mathematica, 3rd ed., Chapman \& Hall/ CRC, Boca Raton, FL, (2006).

11) J. W. Martin and R. D. Doherty: Stability of Microstructure in Metallic Systems, Cambridge University Press, Cambridge, (1976), 179.

12) D. Kammer and P. W. Voorhees: Acta Mater., 54 (2006), 1549.

13) D. A. Drew: SIAM J Appl Math., 50 (1990); 649.

14) D. Hilbert and S. Cohn-Vossen: Anschauliche geometrie, Springer, Berlin, (1932).

15) R. Mendoza, K. Thornton, I. Savin and P. W. Voorhees: Acta Mater., 54 (2006), 743.

16) Y. L. Tian and R. W. Kraft: Metall. Trans. A, 18 (1987), 1359.

17) E. Werner: Acta Metall., 37 (1989), 2047.

18) P. W. Voorhees: J. Status Phys., 38 (1985), 231.

19) E. Werner: Mater. Sci. Eng. A, 132 (1991), 213.

20) R. T. DeHoff: Acta Metall., 43 (1984), 43.

21) P. W. Voorhees, J. Alkemper and R. Mendoza: Adv. Eng. Mater., 4 (2002), 694.

22) R. Mendoza, K. Thornton, I. Savin and P. W. Voorhees: Acta Mater., 54 (2006), 743. 